\title{
Chromatic aberration free reflective mirror-based optical system design for multispectral photoacoustic instruments
}

\author{
Hojong Choi ${ }^{\mathrm{a}}$, Yun Jae Ju ${ }^{\mathrm{b}}$, Jae Heung Jo ${ }^{\mathrm{b}, *}$ and Jae-Myung Ryu ${ }^{\mathrm{c}, *}$ \\ ${ }^{a}$ Department of Medical Convergence Engineering, Kumoh National Institute of Technology, Gumi \\ 39253, Korea \\ ${ }^{\mathrm{b}}$ Department of Photonics and Sensors, Hannam University, Daejon 34430, Korea

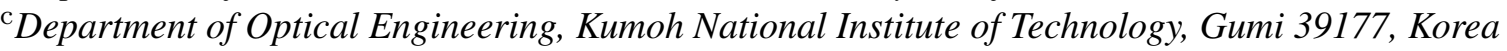

\begin{abstract}
.
BACKGROUND: Current multispectral photoacoustic instruments must use large and separate combinational structures to obtain various biological tissue information for multispectral ranges.

OBJECTIVE: The optical aberration generated from the multispectral photoacoustic systems may reduce the image quality of biological tissue because the improper structures for combining light of different wavelength cannot produce good optical ray convergence points. To prevent this, complex combined structures need to be considered at the design level for multispectral photoacoustic systems.

METHODS: In place of an optical refracted lens system, reflective mirrors could be designed for optical systems. To verify our proposed idea, we assessed optical distortion performance using red, green, and blue light, and combined optical light sources to compare their chromatic aberration characteristics.

RESULTS: The high optical performance is realized regardless of the wavelength for a light source combined with multiple wavelengths, because our optical system was designed with only a reflective surface.

CONCLUSIONS: The designed optical system using a reflective mirror can provide multispectral optical sources (such as infrared, visible, and ultraviolet optical lights) with only one light ray path, without any chromatic aberrations.
\end{abstract}

Keywords: Paraxial design method, reflective mirror system, photoacoustic instruments

\section{Introduction}

Ultrasound systems have been used for various applications such as diagnosis, sound navigation and ranging, and non-destructive areas [1,2]. Optical systems have also been applied in biomedical, mechanical, and telecommunication applications [3-5]. Photoacoustic instruments are combined systems composed of both optical and ultrasound systems. They were recently highlighted for animal and human disease diagnosis because photoacoustic systems utilize the mutual advantages of optical and ultrasound

\footnotetext{
${ }^{*}$ Corresponding authors: Jae Heung Jo, Department of Photonics and Sensors, Hannam University, Daejon 34430, Korea. Tel.: +82 42629 7659; E-mail: jhjo@hnu.kr; Jae-Myung Ryu, Department of Optical Engineering, Kumoh National Institute of Technology, Gumi 39177, Korea. Tel.: +82 54478 7783; E-mail: jmryu@ kumoh.ac.kr.
}

0928-7329/19/\$35.00 (C) 2019 - IOS Press and the authors. All rights reserved

This article is published online with Open Access and distributed under the terms of the Creative Commons Attribution NonCommercial License (CC BY-NC 4.0). 
sources [6]. In photoacoustic instruments, optical light rays generated from the optical systems are transmitted to the desired target, to stimulate thermal expansion [7]. The generated thermal expansion can produce acoustic vibrations from the target, and ultrasound transducers can then detect the acoustic signals to obtain the target information $[8,9]$. The optical systems can provide higher contrast and lower spatial resolution for organic soft tissues, compared to the use of ultrasound-only systems [10]. Ultrasound systems typically provide lower contrast, and higher spatial resolutions and penetration depths, compared to optical-only systems [11]. For photoacoustic instruments, the light source power is lower than ultrasound power, such that the sensitivities of the photoacoustic instruments are typically lower [8]. Optical sources can provide biological, chemical, and physiological information, such as oxyhemoglobin, deoxyhemoglobin, lipid, melanin, collagen, and elastin information, and ultrasound sources can determine structural information, such as blood vessel wall, fat, and blood velocity [7].

Photoacoustic instruments can be divided into two categories: optical resolution- and acoustic resolution-based photoacoustic instruments [12]. For optical resolution-based photoacoustic instruments, focused ultrasound transducers (either singly or in an array) with mechanical motors are used to scan the organic tissue [13]. For acoustic resolution based-photoacoustic instruments, focused light is used to obtain the target information [7]. The image resolution of optical resolution- and acoustic resolution-based photoacoustic instruments primarily depends on the optical resolution of the optical systems and spatial resolution of the ultrasound transducers [14]. Therefore, optical resolution basedphotoacoustic instruments provide higher optical contrast resolution at reduced penetration depths than that of the acoustic resolution based-photoacoustic instruments [15]. However, both photoacoustic instruments need to utilize well-designed optical systems to obtain high quality functional, anatomical, and biological information from the soft tissues of animals and human beings.

Each light used as an optical source has its own limited wavelength range [8]. Therefore, each light can generate different biological tissue information due to different penetration depths. For instance, visible light in the red, green, and blue ranges (between 380 and $740 \mathrm{~nm}$ ), can be absorbed in certain hemoglobin and melanin in the tissue [8]. The photoacoustic instruments using only a single wavelength light can provide the specific biological tissue data because the protein, melanin, and collagen have different absorption coefficient. Therefore, multispectral photoacoustic instruments were recently implemented to obtain a variety of biological data simultaneously [16-18]. To utilize multispectral light for obtaining a variety of biological data, photoacoustic instruments have been constructed, either with a variety of combinational structures or with multi optical fibers [16]. However, this method uses the lens to construct the optical system through the refraction of light. In this case, the refractive index of the material used for the lens changes depending on the wavelength. Therefore, when a refractive optical system is used, chromatic aberration is always generated [16,17]. To reduce the optical chromatic aberrations generated when combining the light beam, the designed convergence structures require detailed optical ray convergence points with proper light power levels $[19,20]$. Therefore, we designed novel optical system structures that utilize only one light ray path, to avoid light convergence problems when generating the transmitted light beam for photoacoustic instruments. To the best of our knowledge, we are the first to design a chromatic aberration-free optical system using reflective mirror structures for photoacoustic instruments. The low optical aberration in these optical systems generates low optical resolution for the photoacoustic instruments. In particular, multispectral photoacoustic instruments must generate broad spectral ranges to obtain the desired sample information when combining light of different wavelengths $[21,22]$. Therefore, the proposed scheme could be one solution for solving the optical chromatic aberration problems for current multispectral photoacoustic instruments. Section 2 describes the detailed optical design methods of the chromatic aberration-free optical system. Section 3 shows the results and discussion of the designed optical systems and a comparison of the illumination intensity of the combined light beam sources. Section 4 provides concluding remarks. 


\section{Paraxial design}

Optical systems with very narrow field angles, such as telescopes, often consist of only reflective surfaces [23]. However, in the systems, it is very difficult to construct an optical system with only a reflective surface, because the field angle is very wide for photoacoustic instruments [24]. However, optical systems are essentially similar to wide-angle systems, such as in fish-eye optics. The negativelead type means that the focal length of the first element of the optical system is negative. Such a negative-lead type optical system has a shorter effective focal length (EFL) than back focal length (BFL) and is mainly applied to an optical system with a wide angle of view [25]. Therefore, this optical system must be designed with both a concave and convex mirror. Because the refractive power of the mirror is determined by $k=-2 / R$, the image height and EFL of the optical system consisting of two mirrors are given by Eqs (1) and (2).

$$
\begin{aligned}
& {\left[k_{1},-z_{1}, k_{2},-z_{2}\right]=\left[-\frac{2}{R_{1}},-z_{1},-\frac{2}{R_{2}},-z_{2}\right]=0} \\
& {\left[k_{1},-z_{1}, k_{2}\right]=\left[-\frac{2}{R_{1}},-z_{1},-\frac{2}{R_{2}}\right]=\frac{1}{f}}
\end{aligned}
$$

where the suffix denotes the surface number, $R$ is the radius of curvature of the mirror, $z$ is the spacing of the surface, and $\mathrm{f}$ is the EFL of the optical system.

Here, the total number of unknowns is five; hence, at least three conditions must be provided. In general photoacoustic imaging systems, it is very important to determine the desired target shapes for the optimal mirror and lens, because the chromatic aberration changes rapidly depending on their shapes [26]. However, an optical illumination system is required regardless of the wavelength of the light and the position of the target samples. Therefore, the optical system is initially assumed to be a Cassegrain reflective system, and the shape of the two mirrors is determined as an arbitrary value [27]. In the reflective system, an axial ray at the primary mirror is shielded by the secondary mirror; hence, the primary mirror must be larger than the secondary mirror. Therefore, $R_{1}$ (the radius of the primary mirror) $>R_{2}$ (the radius of the secondary mirror) must be satisfied. In this study, $R_{1}=110 \mathrm{~mm}$ and $R_{2}=34.5 \mathrm{~mm}$. Suppose that the optical system is designed to exhibit equisolid distortion, and that the field angle range is from $40^{\circ}$ to $110^{\circ}$. Further, let us suppose that the image height at $110^{\circ}$ is $14.5 \mathrm{~mm}$. An instrument body containing an image sensor with a half-diagonal size of $14.5 \mathrm{~mm}$ is easily available. Therefore, if the specifications are determined and the optical system is determined, the performance of the optical system can be easily confirmed.

In an optical system with equisolid distortion, the relationship between the field angle $\theta$, the halfdiagonal size $Y$, and the EFL $f$, is given by Eq. (3).

$$
Y=2 f \sin \frac{\theta}{2}
$$

Under such conditions, the focal length of this optical system is found to be approximately $8.8 \mathrm{~mm}$, and the optical path is shown in Fig. 1.

If designed as shown in Fig. 1, the light source should be placed on the image plane. However, this is not possible due to mechanical interference. Therefore, this must be considered during the design of our relay system. In the relay system, the image formed by the optical system shown in Fig. 1 should be reduced to the size of the light emitting diode (LED). However, if the reduction magnification is high, many mirrors should be used for optical aberration correction. To homogenize the light from the LED, a glass rod with an entrance and exit surface area ratio of four should be used. The most easily 


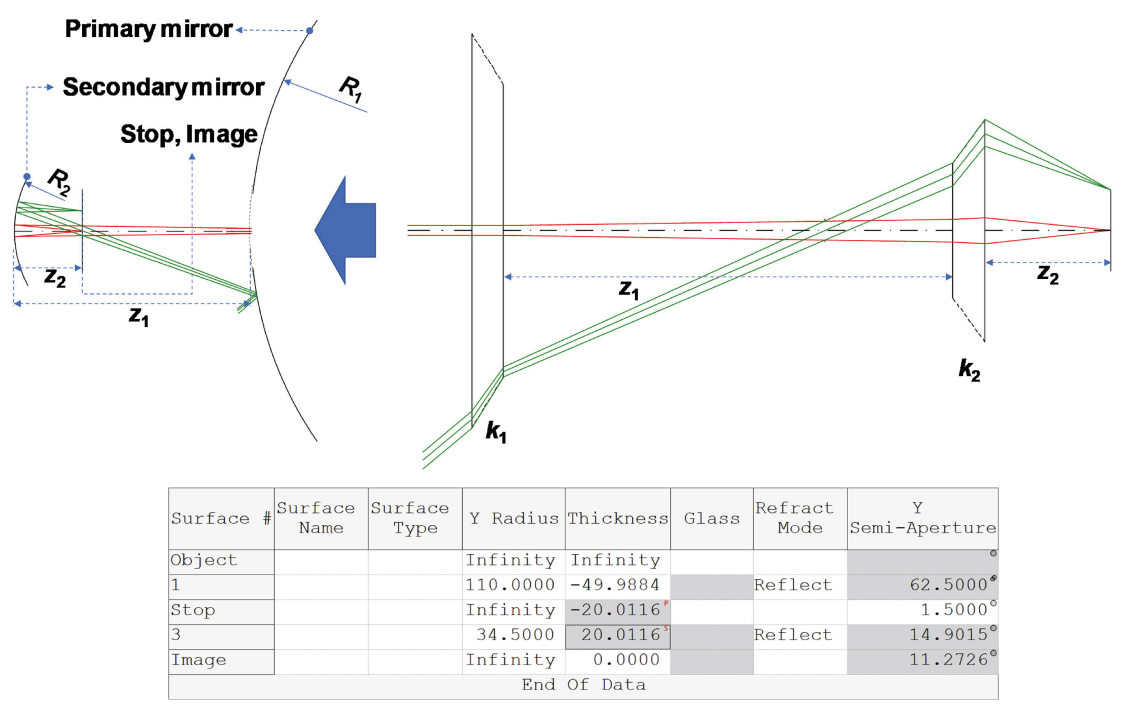

Fig. 1. Optical layout of paraxial design for wide-angle system.

available and inexpensive glass rod has an entrance aperture of $6.25 \mathrm{~mm}^{2}$ and an exit aperture of $25 \mathrm{~mm}^{2}$. Positioning the LED in the entrance aperture of the glass rod reduces the numerical aperture (NA) at the exit aperture. In this case, the number of mirrors used in the optical system can be reduced. The halfdiagonal size of the glass rod with an exit aperture of $25 \mathrm{~mm}^{2}$ and a square cross section is approximately $3.54 \mathrm{~mm}$. The resulting magnification of the relay system is approximately -0.244 because the image height of the optical system, as shown in Fig. 1, is $14.5 \mathrm{~mm}$. If we suppose the relay system also uses two mirrors, then the axial ray height in the image plane and the magnification would be determined by Eqs (4) and (5).

$$
\begin{aligned}
& {\left[-z_{3},-\frac{2}{R_{3}},-z_{4},-\frac{2}{R_{4}},-z_{5}\right]=0} \\
& {\left[-z_{3},-\frac{2}{R_{3}},-z_{4},-\frac{2}{R_{4}}\right]=m}
\end{aligned}
$$

In Eqs (4) and (5), $m$ is the magnification and $m=-0.244$. Therefore, there are five unknowns, and three additional conditions must be provided. The distance between the mirrors will be approximately determined. Assuming that the distance $z_{3}$ from the image surface of the optical system to the first mirror of the relay system is $90 \mathrm{~mm}$, the distance $z_{4}$ between the two mirrors is $50 \mathrm{~mm}$, and the distance $z_{5}$ from the last mirror to the image surface is $160 \mathrm{~mm}$, and $R_{3}=30.06$ and $R_{4}=90.992$ are obtained. The optical layout of the relay system is shown in Fig. 2. Therefore, a paraxial design can be obtained by combining two designed optical systems.

\section{Results and discussion}

\subsection{Optimization process}

In general, the paraxial design is the result of determining the initial shape and spacing of the lens or mirror [28]. Therefore, the target performance is barely obtained. To correct this, the light beam 


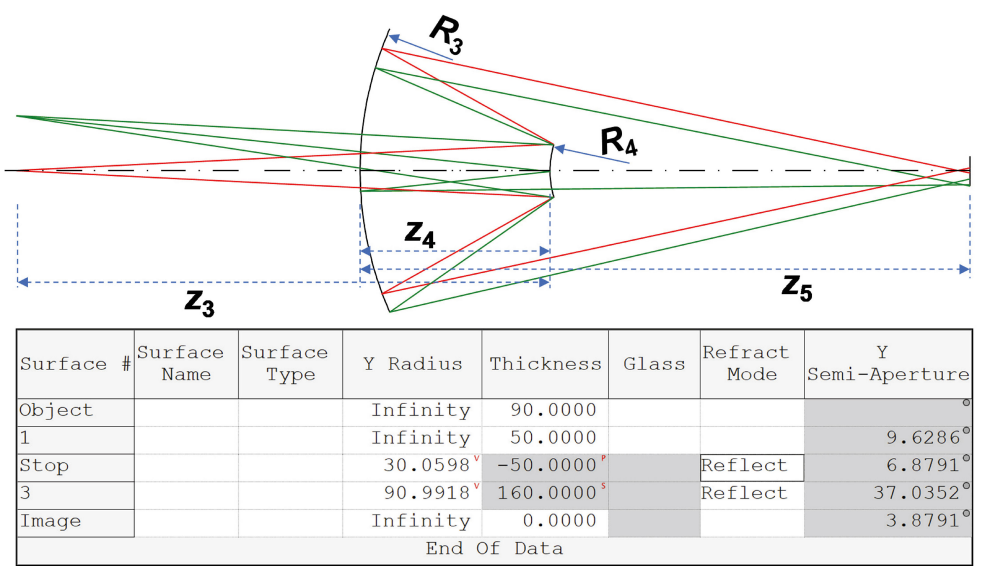

Fig. 2. Optical layout for relay system.

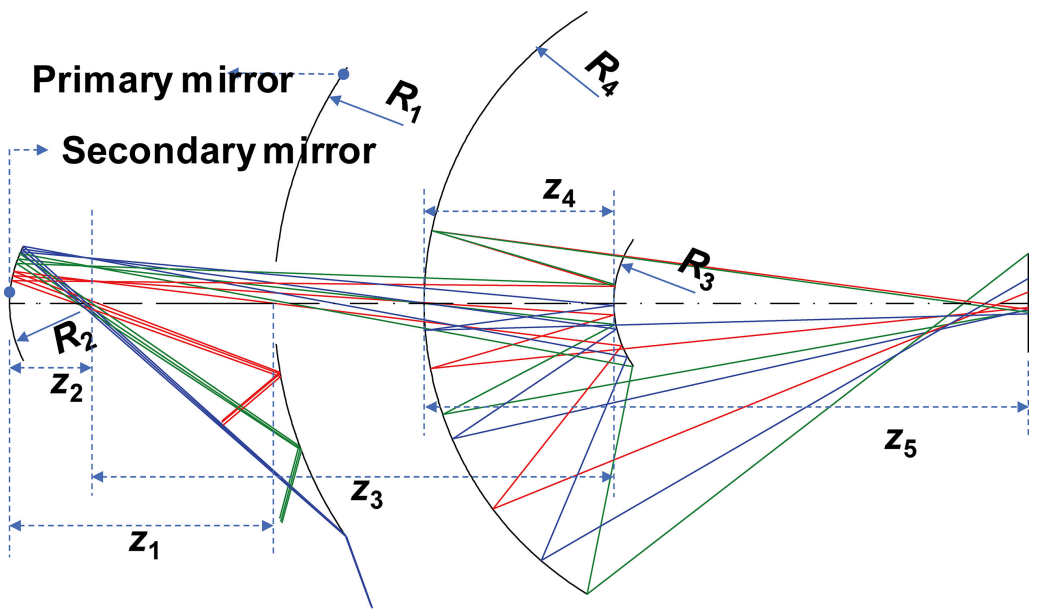

(a) Optical layout of our paraxial design.

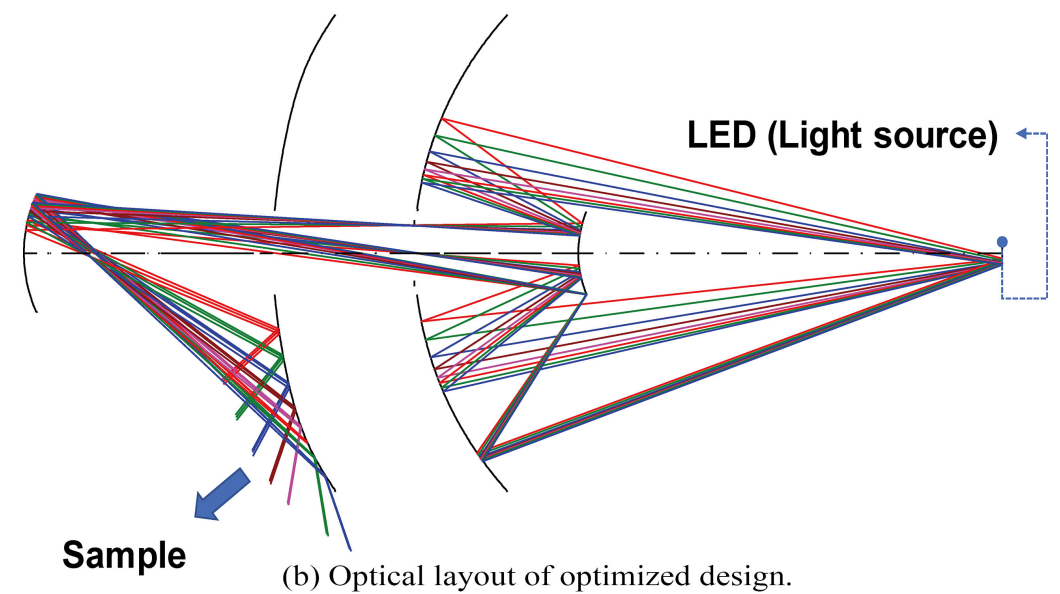

Fig. 3. Optical layout for coupled system. 

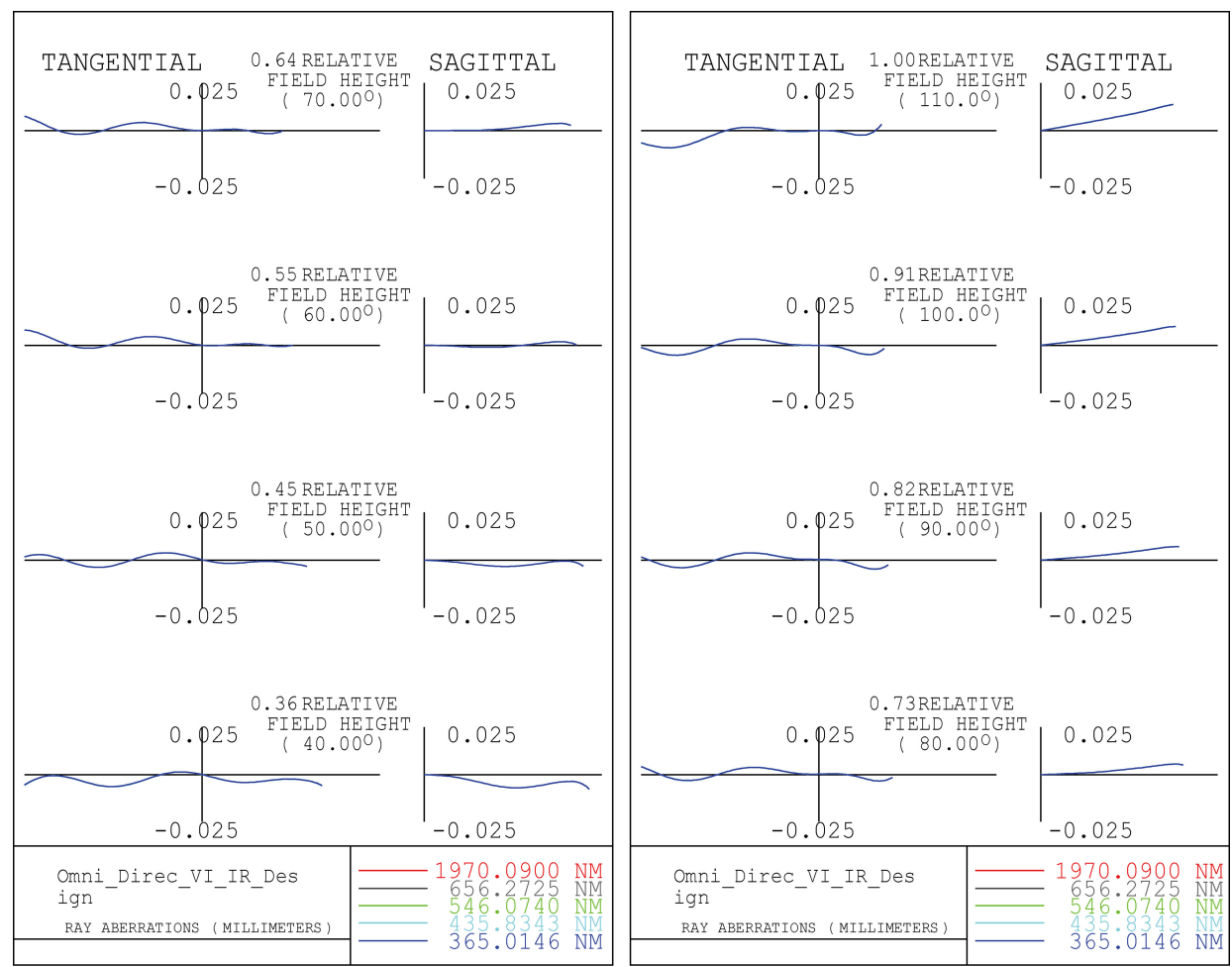

Fig. 4. Ray fan of our optimized system.

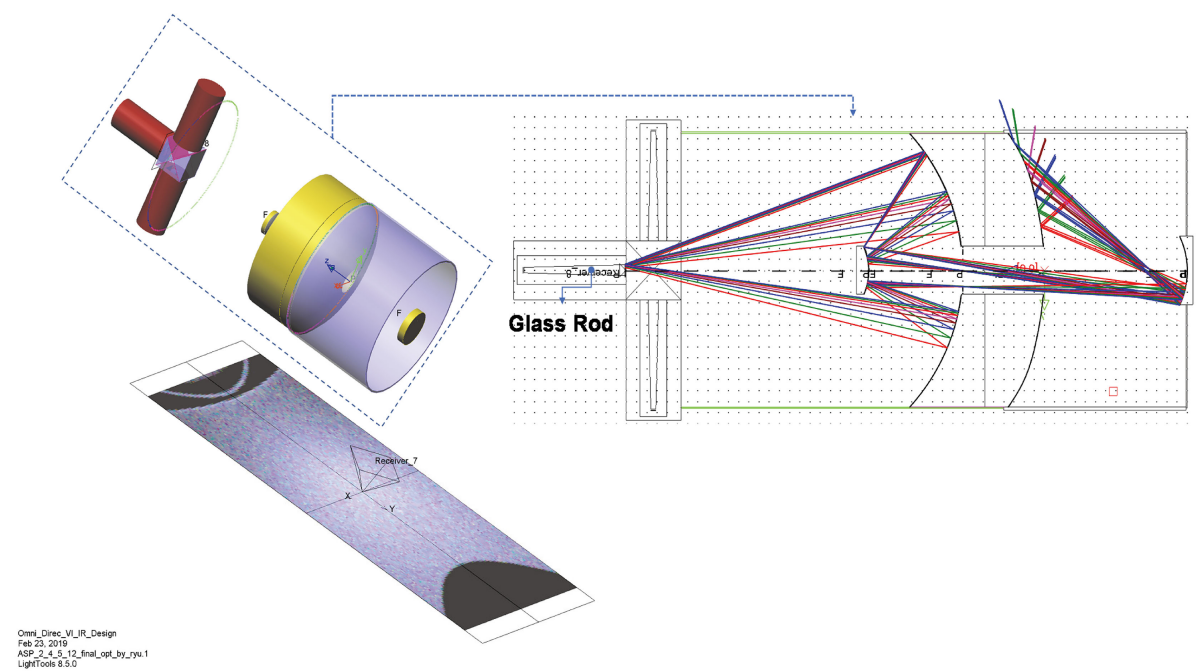

Fig. 5. 3D optical layout for our optimized system.

should be focused while changing the shape and spacing of the mirror gradually. In acoustic-resolutionbased photoacoustic instruments, the light beam needs to be focused. Simultaneously, the ultrasonic transducers need to be covered to obtain the target information. On the other hand, a light ray passing through the optical system forms a spot on the image surface. Optimization in optical design means 
ASP_2_4_5_12_final_opt_by_ryu.1 Receiver_7 Forward Simulation Irradiance, $\mathrm{W} / \mathrm{mm}^{\wedge} 2$

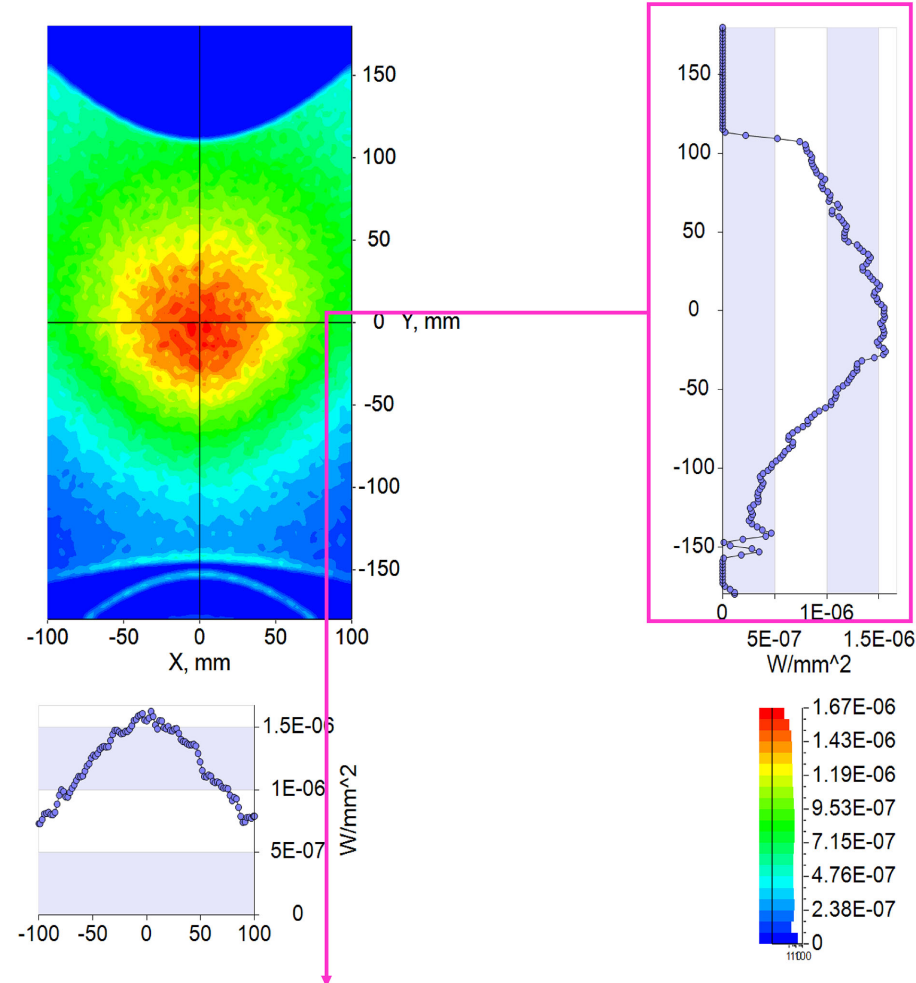

The normalized intensity profile at $\mathrm{Y}$-axis

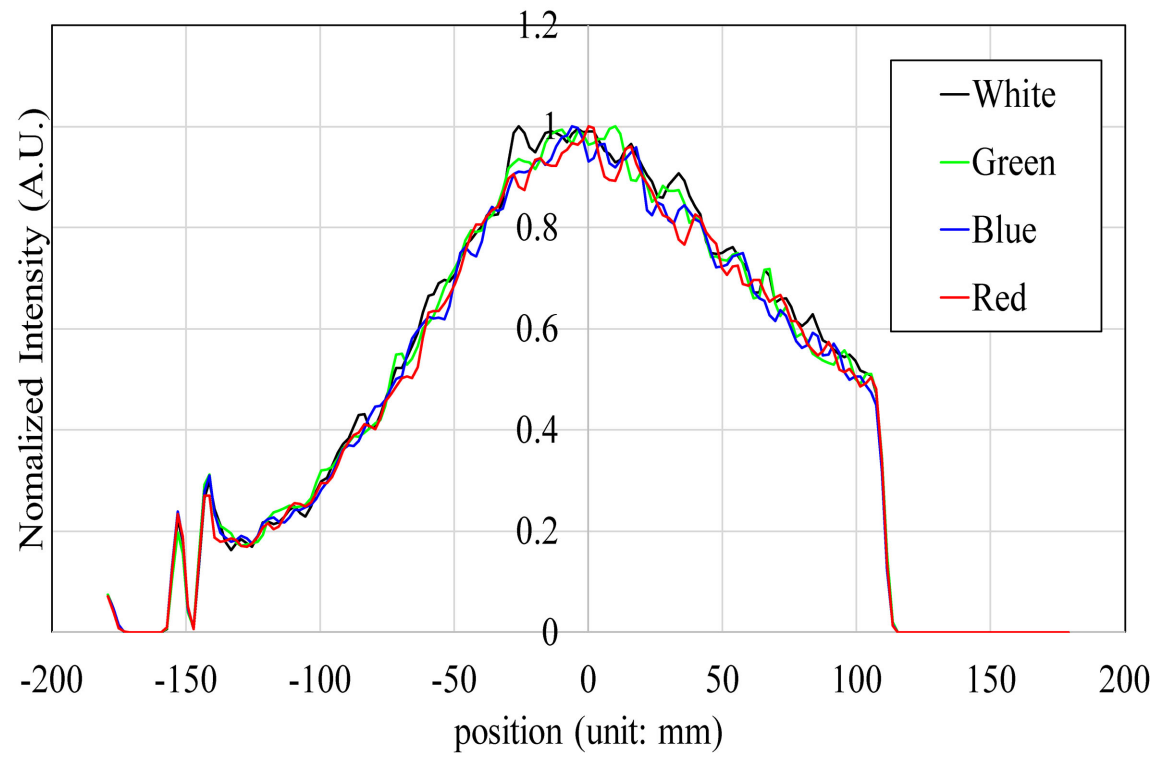

Fig. 6. Intensity distribution and normalized intensity profile. 
minimizing the size of these spots. The spot size is a function of the curvature and spacing of the mirror, and optimization is the process of solving the nonlinear simultaneous equations for the spot size. The optical path of the optimized optical system and the paraxial design determined in Section 2 are shown in Fig. 3. This process is meaningful because low chromatic aberration within the target area could deteriorate the resolution in the photoacoustic instruments. As shown in Fig. 3, because the optical system consists only of the reflective surface, there is no change in the focus position as a result of the wavelength. Therefore, it is useful to generate high resolution with low chromatic aberrations in the photoacoustic instruments.

\subsection{Performance verification}

The developed multispectral photoacoustic instruments have high chromatic aberrations when using the LED lights with multiple wavelength [16,29]. However, this designed optical system can be expected to have no chromatic aberration because there is no change in focal position with respect to wavelength. This is highly desirable. In fact, Fig. 4 shows a ray fan in optical design software. As shown in Fig. 4, it can be seen that there is no chromatic aberration from the infrared (IR) region to the ultraviolet region.

On the other hand, by combining LEDs with these optical systems, we confirmed the intensity distribution of light at specific locations. An X-cube prism may be required to use LEDs of various wavelengths. The optical system with an LED and a glass rod is shown in Fig. 5.

The intensity distribution at a position $150 \mathrm{~mm}$ away from the optical axis in the vertical direction is shown in Fig. 6. The figure below shows the normalization based on the maximum at each wavelength, and the profile is almost constant regardless of the wavelength. Since the optical system without chromatic aberration is used, it is possible to obtain the same intensity distribution of light by using X-cube and glass rod and synthesizing light sources of different wavelengths. Simulation results show that the region with the strongest light intensity is within $80 \mathrm{~mm}$ in diameter from the center position of the sample. Adequate intensity distributions within a $150 \mathrm{~mm}$ range for red, green, and blue LED lights are enough to cover the samples for the photoacoustic instruments.

\section{Conclusion}

A chromatic aberration-free optical system using reflective mirrors was designed for multispectral photoacoustic instruments. The LED light sources are cost-effective and relatively safe compared to the traditional laser light sources used in photoacoustic instruments. Therefore, the photoacoustic instruments using LED light sources have been designed. Because different wavelengths are needed for different tissue characterization, multi-spectral photoacoustic instruments have been developed. However, the currently developed multispectral photoacoustic instruments have high chromatic aberrations, thus deteriorating optical resolution. Therefore, we developed a new type of multispectral optical design using a reflective mirror instead of the refracted optical lens used in conventional systems. Because we constructed a system with only a reflective surface, an aberration-free optical system was achieved. These results were confirmed from aberration plots and a normalized intensity profile. In the optical design, the intensity distribution at a position $150 \mathrm{~mm}$ away from the optical axis in the vertical direction exhibited adequate light intensity values. Therefore, the developed chromatic aberration-free optical system using a reflective mirror is a potential candidate for use in multispectral photoacoustic instruments. 


\section{Acknowledgments}

This research was supported by the Basic Science Research Program through the National Research Foundation of Korea (NRF) funded by the Ministry of Science, ICT and Future Planning (NRF2017R1C1B1003606) and the Ministry of Education (NRF-2017R1D1A3B03029119).

\section{Conflict of interest}

None to report.

\section{References}

[1] He Z. Optimization of acoustic emitted field of transducer array for ultrasound imaging. Bio-Med Mater Eng. 2014; 24(1): 1201.

[2] Shung KK. Diagnostic Ultrasound: Imaging and Blood Flow Measurements. Boca Raton, FL, USA: Taylor \& Francis; 2015.

[3] Kaminow I, Li T, Willner AE. Optical Fiber Telecommunications VB: Systems and Networks. Amsterdam, Netherlands: Elsevier; 2010.

[4] Lee J-H, Kim YN, Park H-J. Bio-optics based sensation imaging for breast tumor detection using tissue characterization. Sensors. 2015; 15(3): 6306-23.

[5] Lee J, Sevag Packard RR, Xu H, Kang H, Baek KI, Jen N, Jay Kuo C, Chi NC, Ho C-M, Li R. Light-sheet microscopy to elucidate hemodynamic forces and modulation of cardiac trabeculation: implications for embryonic contractile function. J Clin Invest. 2016; 30(1_supplement): 554.3-.3.

[6] Emelianov SY, Li P-C, O’Donnell M. Photoacoustics for molecular imaging and therapy. Phys Today. 2009; 62(8): 34-9.

[7] Wang LV. Photoacoustic Imaging and Spectroscopy. Boca Raton, FL, USA: CRC press; 2009.

[8] Beard P. Biomedical photoacoustic imaging. Interface Focus. 2011; 1(4): 602-31.

[9] Jeong JJ, Choi H. An impedance measurement system for piezoelectric array element transducers. Measurement. 2017; 97: 138-44.

[10] Li X, Wei W, Zhou Q, Shung KK, Chen Z. Intravascular photoacoustic imaging at 35 and 80 MHz. J. Biomed. Opt. 2012; 17(10): 106005.

[11] Zhu B, Xu J, Li Y, Wang T, Xiong K, Lee C, Yang X, Shiiba M, Takeuchi S, Zhou Q. Micro-particle manipulation by single beam acoustic tweezers based on hydrothermal PZT thick film. AIP Adv. 2016; 6(3): 035102.

[12] Choi H, Ryu J, Kim J. A novel fisheye-lens-based photoacoustic system. Sensors. 2016; 16(12): 2185.

[13] Li L, Yeh C, Hu S, Wang L, Soetikno BT, Chen R, Zhou Q, Shung KK, Maslov KI, Wang LV. Fully motorized opticalresolution photoacoustic microscopy. Opt Lett. 2014; 39(7): 2117-20.

[14] Zhou Y, Yao J, Wang LV. Tutorial on photoacoustic tomography. J. Biomed. Opt. 2016; 21(6): 061007.

[15] Lin L, Zhang P, Xu S, Shi J, Li L, Yao J, Wang L, Zou J, Wang LV. Handheld optical-resolution photoacoustic microscopy. J. Biomed. Opt. 2016; 22(4): 041002.

[16] Cao R, Kilroy JP, Ning B, Wang T, Hossack JA, Hu S. Multispectral photoacoustic microscopy based on an opticalacoustic objective. Photoacoustics. 2015; 3(2): 55-9.

[17] Razansky D, Distel M, Vinegoni C, Ma R, Perrimon N, Koster RW, Ntziachristos V. Multispectral opto-acoustic tomography of deep-seated fluorescent proteins in vivo. Nat Photon. 2009; 3(7): 412-7.

[18] Yao J, Wang LV. Photoacoustic microscopy. Laser Photonics Rev. 2013; 7(5): 758-78.

[19] Choi H, Ryu J-M, Yeom J-Y. Development of a double-gauss lens based setup for optoacoustic applications. Sensors. 2017; 17(3): 496.

[20] Jenkins FA, White HE. Fundamentals of Optics. Noida, India: Tata McGraw-Hill Education; 1957.

[21] Choi H, Yeom J-Y, Ryu J-M. Development of a multiwavelength visible-range-supported opto-ultrasound instrument using a light-emitting diode and ultrasound transducer. Sensors. 2018; 18(10): 3324.

[22] Zappe H. Fundamentals of Micro-optics. Cambridge, United Kingdom: Cambridge University Press; 2010.

[23] Pedrotti FL, Pedrotti LS. Introduction to Optics. Englewood Cliffs, NJ, USA: Prentice-Hall 1993.

[24] Choi H, Ryu J, Yeom J-Y. A cost-effective light emitting diode-acoustic system for preclinical ocular applications. Curr Op Photon. 2018; 2(1): 59-68.

[25] Walker BH. Optical Design for Visual Systems. Bellingham, WA, USA: SPIE Press; 2000. 
[26] Mahajan VN. Optical Imaging and Aberrations: Part I: Ray Geometrical Optics. Bellingham, WA USA: SPIE Press; 1998.

[27] Smith WJ. Modern Optical Engineering. New York, NY, USA: McGraw-Hill Education; 2007.

[28] Kidger MJ. Intermediate Optical Design. Bellingham, WA, USA: SPIE Publications; 2004.

[29] Adachi Y, Hoshimiya T. Photoacoustic imaging with multiple-wavelength light-emitting diodes. Jpn J Appl Phys. 2013; 52(7S): 07HB6. 